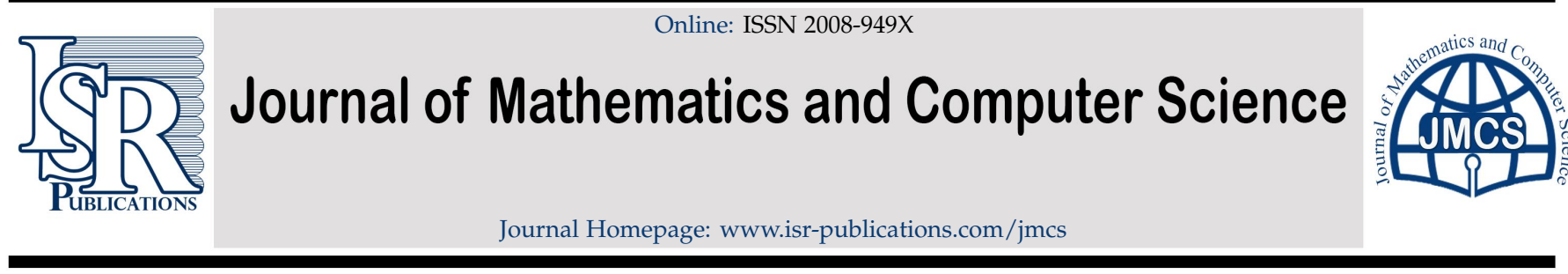

\title{
Expressions and dynamical behavior of solutions of a class of rational difference equations of fifteenth-order
}

\author{
A. M. Ahmed ${ }^{a, b, *}$, Samir Al Mohammady ${ }^{a, c}$, Lama Sh. Aljoufia \\ a Department of Mathematics, College of Science, Jouf University, P.O. Box 2014, Sakaka, Jouf, Saudi Arabia. \\ ${ }^{b}$ Department of Mathematics, Faculty of Science, Al Azhar University, Nasr City 11884, Cairo, Egypt. \\ ${ }^{c}$ Department of Mathematics, Faculty of Science, Helwan University, Helwan 11795, Egypt.
}

\begin{abstract}
The main goal of this paper, is to obtain the forms of the solutions of the following nonlinear fifteenth-order difference equations

$$
x_{n+1}=\frac{x_{n-14}}{ \pm 1 \pm x_{n-2} x_{n-5} x_{n-8} x_{n-11} x_{n-14}}, \quad n=0,1,2, \ldots,
$$

where the initial conditions $x_{-14}, x_{-13}, \ldots, x_{0}$ are arbitrary real numbers. Moreover, we investigate stability, boundedness, oscillation and the periodic character of these solutions. Finally, we confirm the results with some numerical examples and graphs by using Matlab program.
\end{abstract}

Keywords: Recursive sequence, oscillation, semicycles, stability, periodicity, solutions of difference equations.

2020 MSC: 39A10, 39A22, 39A23.

(C)2022 All rights reserved.

\section{Introduction}

Difference equations appear as natural descriptions of observed evolution phenomena because most measurements of time evolving variables are discrete and as such these equations are in their own right important mathematical models. More importantly, difference equations also appear in the study of discretization methods for differential equations. Several results in the theory of difference equations have been obtained as more or less natural discrete analogues of corresponding results of differential equations.

Recently there has been a lot of interest in studying the global attractivity, boundedness character, periodicity and the solution form of nonlinear difference equations. For some results in this area, for example, Ahmed et al. [5] obtained the expressions of solutions of the class of difference equations

$$
x_{n+1}=\frac{x_{n-2 k+1}}{ \pm 1 \pm \prod_{i=1}^{k} x_{n-2 i+1}}, \quad n=0,1,2, \ldots,
$$

\footnotetext{
*Corresponding author

Email addresses: ahmedelkb@yahoo.com \& amaahmed@ju.edu.sa (A. M. Ahmed), senssar@ju.edu.sa \&

drsamirhnk@gmail .com (Samir Al Mohammady), lamashuja11@gmail.com (Lama Sh. Aljoufi)

doi: $10.22436 /$ jmcs.025.01.02
}

Received: 2021-01-28 Revised: 2021-02-24 Accepted: 2021-03-18 
with conditions posed on the initial values $x_{-j}, j=0,1,2, \ldots, 2 k-1$, where $k \in\{1,2, \ldots\}$. Elsayed et al [13] obtained the solutions of the difference equations

$$
x_{n+1}=\frac{x_{n-11}}{ \pm 1 \pm x_{n-2} x_{n-5} x_{n-8} x_{n-11}}, \quad n=0,1,2, \ldots,
$$

where the initial conditions are arbitrary real numbers. Cinar [8-10] obtained the solutions of the difference equations

$$
x_{n+1}=\frac{x_{n-1}}{1+x_{n} x_{n-1}}, \quad x_{n+1}=\frac{x_{n-1}}{-1+x_{n} x_{n-1}}, \quad x_{n+1}=\frac{a x_{n-1}}{1+b x_{n} x_{n-1}} .
$$

Elsayed [12] studied the difference equation

$$
x_{n+1}=\frac{x_{n-5}}{-1+x_{n-2} x_{n-5}} .
$$

For other related papers, see [1-4, 6, 7, 11, 14-17].

In this paper, we obtain the solutions of the following nonlinear difference equations

$$
x_{n+1}=\frac{x_{n-14}}{ \pm 1 \pm x_{n-2} x_{n-5} x_{n-8} x_{n-11} x_{n-14}}, \quad n=0,1,2, \ldots,
$$

with conditions posed on the initial values $x_{-j}, j=0,1,2, \ldots, 14$. Moreover, we investigate stability, boundedness, oscillation and the periodic character of these solutions. Finally, we confirm the results with some numerical examples and graphs by using Matlab program.

Let I be some interval of real numbers and let

$$
\mathrm{F}: \mathrm{I}^{\mathrm{k}+1} \rightarrow \mathrm{I},
$$

be a continuously differentiable function. Then for every set of initial conditions $x_{-k}, x_{-k+1}, \ldots, x_{0} \in I$, the difference equation

$$
x_{n+1}=F\left(x_{n}, x_{n-1}, x_{n-2}, \ldots, x_{n-k}\right), \quad n=0,1, \ldots,
$$

has a unique solution $\left\{x_{\mathfrak{n}}\right\}_{\mathfrak{n}=-k}^{\infty}$.

Definition 1.1. A point $\bar{x} \in \mathrm{I}$ is called an equilibrium point of Eq. (1.1) if

$$
\bar{x}=F(\bar{x}, \bar{x}, \ldots, \bar{x}),
$$

that is,

$$
x_{n}=\bar{x} \text { for all } n \geqslant-k
$$

is a solution of Eq. (1.1), or equivalently, $\bar{x}$ is a fixed point of $G(x)=F(x, \ldots, x)$.

Definition 1.2. A sequence $\left\{x_{n}\right\}_{n=-k}^{\infty}$ is said to be periodic with period $p$ if $x_{n+p}=x_{n}$ for all $n \geqslant-k$.

\section{Definition 1.3.}

(i) The equilibrium point $\bar{x}$ of Eq. (1.1) is locally stable if for every $\epsilon>0$, there exists $\delta>0$ such that for all $x_{-k}, x_{-k+1}, \ldots, x_{-1}, x_{0} \in$ I with

$$
\left|x_{-k}-\bar{x}\right|+\left|x_{-k+1}-\bar{x}\right|+\cdots+\left|x_{0}-\bar{x}\right|<\delta,
$$

we have

$$
\left|x_{n}-\bar{x}\right|<\epsilon \text { for all } n \geqslant-k
$$


(ii) The equilibrium point $\bar{x}$ of Eq. (1.1) is locally asymptotically stable if $\bar{x}$ is locally stable of Eq. (1.1) and there exists $\gamma>0$, such that for all $x_{-k}, x_{-k+1}, \ldots, x_{-1}, x_{0} \in I$ with

$$
\left|x_{-k}-\bar{x}\right|+\left|x_{-k+1}-\bar{x}\right|+\cdots+\left|x_{0}-\bar{x}\right|<\gamma,
$$

we have

$$
\lim _{n \rightarrow \infty} x_{n}=\bar{x}
$$

(iii) The equilibrium point $\bar{x}$ of Eq. (1.1) is global attractor if for all $x_{-k}, x_{-k+1}, \ldots, x_{-1}, x_{0} \in I$, we have

$$
\lim _{n \rightarrow \infty} x_{n}=\bar{x} .
$$

(iv) The equilibrium point $\bar{x}$ of Eq. (1.1) is globally asymptotically stable if $\bar{x}$ is locally stable, and $\bar{x}$ is also a global attractor of Eq. (1.1).

(v) The equilibrium point $\bar{x}$ of Eq. (1.1) is unstable if $\bar{x}$ is not locally stable.

\section{Definition 1.4.}

(i) A sequence $\left\{x_{n}\right\}$ is said to oscillate about zero or simply to oscillate if the terms $x_{n}$ are neither eventually all positive nor eventually all negative. Otherwise the sequence is called nonoscillatory. A sequence $\left\{x_{n}\right\}$ is called strictly oscillatory if for every $n_{0} \geqslant 0$, there exist $n_{1}, n_{2} \geqslant n_{0}$ such that $x_{n_{1}} x_{n_{2}}<0$.

(ii) A sequence $\left\{x_{n}\right\}$ is said to oscillate about $\bar{x}$ if the sequence $\left\{x_{n}-\bar{x}\right\}$ oscillates. The sequence $\left\{x_{n}\right\}$ is called strictly oscillatory about $\bar{x}$ if the sequence $\left\{x_{n}-\bar{x}\right\}$ is strictly oscillatory.

Definition 1.5. A positive semicycle of a solution $\left\{x_{n}\right\}$ of Eq. (1.1) consists of a "string" of terms $\left\{x_{l}, x_{l+1}, \ldots, x_{m}\right\}$, all greater than or equal to $\bar{x}$, with $l \geqslant-k$ and $m \leqslant \infty$ and such that

$$
\text { either } l=-k \text { or } l>-k \text { and } x_{l-1}<\bar{x},
$$

and

$$
\text { either } m=\infty \text { or } m<\infty \text { and } x_{m+1}<\bar{x} \text {. }
$$

A negative semicycle of a solution $\left\{x_{n}\right\}$ of Eq. (1.1) consists of a "string" of terms $\left\{x_{l}, x_{l+1}, \ldots, x_{m}\right\}$, all less than $\bar{x}$, with $l \geqslant-k$ and $m \leqslant \infty$ and such that

$$
\text { either } l=-k \text { or } l>-k \text { and } x_{l-1} \geqslant \bar{x},
$$

and

$$
\text { either } m=\infty \text { or } m<\infty \text { and } x_{m+1} \geqslant \bar{x} \text {. }
$$

Definition 1.6. Suppose that the function $F$ is continuously differentiable in some open neighborhood of an equilibrium point $\bar{x}$. Let

$$
p_{i}=\frac{\partial F}{\partial u_{i}}(\bar{x}, \bar{x}, \ldots, \bar{x}) \quad \text { for } i=0,1, \ldots, k,
$$

denote the partial derivatives of $F\left(\mathfrak{u}_{0}, \mathfrak{u}_{1}, \ldots, \mathfrak{u}_{k}\right)$ evaluated at the equilibrium $\bar{x}$ of Eq. (1.1). Then the equation

$$
y_{n+1}=p_{0} y_{n}+p_{1} y_{n-1}+\cdots+p_{k} y_{n-k}, \quad n=0,1, \ldots,
$$

is called the linearized equation associated of Eq. (1.1) about the equilibrium point $\bar{x}$ and the equation

$$
\lambda^{k+1}-p_{0} \lambda^{k}-\cdots-p_{k-1} \lambda-p_{k}=0,
$$

is called the characteristic equation of Eq. (1.2) about $\bar{x}$.

The following result known as the linearized stability theorem is very useful in determining the local stability character of the equilibrium point $\bar{x}$ of Eq. (1.1). 
Definition 1.7. Let $\bar{x}$ be an equilibrium of Eq. (1.1).

(i) $\bar{x}$ is called a hyperbolic equilibrium if Eq. (1.3) has no roots with absolute value equal to one.

(ii) $\bar{x}$ is called a nonhyperbolic equilibrium if there exists a root of Eq. (1.3) with absolute value equal to one.

Throughout this paper, we define $\bmod (k, 3)=k-3\left[\frac{k}{3}\right]$, where $[x]$ be the greatest integer less than or equal to the real number $x$.

\section{The difference equation $x_{n+1}=\frac{x_{n-14}}{1+x_{n-2} x_{n-5} x_{n-8} x_{n-11} x_{n-14}}$}

In this section we give a specific form of the solutions of the first equation in the form

$$
x_{n+1}=\frac{x_{n-14}}{1+x_{n-2} x_{n-5} x_{n-8} x_{n-11} x_{n-14}}, \quad n=0,1,2, \ldots,
$$

with conditions posed on the initial values $x_{-j}, j=0,1,2, \ldots, 14$. Also, we investigate the stability and boundedness of these solutions.

Theorem 2.1. Let $\left\{x_{n}\right\}_{n=-14}^{\infty}$ be a solution of the difference Eq. (2.1). Then for $n=0,1,2, \ldots$,

$$
x_{15 n-k}=a_{k} \prod_{i=0}^{n-1}\left(\frac{1+\left(5 i+M_{k}-1\right) P_{k}}{1+\left(5 i+M_{k}\right) P_{k}}\right),
$$

where $\mathrm{P}_{\mathrm{k}}=\prod_{j=0}^{4} \mathrm{a}_{\bmod (\mathrm{k}, 3)+3 j}, \mathrm{M}_{\mathrm{k}}=5-\left[\frac{\mathrm{k}}{3}\right]$ and $\mathrm{x}_{-\mathrm{k}}=\mathrm{a}_{\mathrm{k}}$, with $\mathrm{rP}_{\mathrm{k}} \neq-1$ such that $\mathrm{r} \in\{1,2,3, \ldots\}, \mathrm{k}=$ $0,1,2, \ldots, 14$.

Proof. For $n=0$, the result holds. Now suppose that $n>0$ and that our assumption holds for $n-1$. That is

$$
x_{15 n-15-k}=a_{k} \prod_{i=0}^{n-2}\left(\frac{1+\left(5 i+M_{k}-1\right) P_{k}}{1+\left(5 i+M_{k}\right) P_{k}}\right) .
$$

Now, it follows from Eq. (2.1) and using Eq. (2.3) that

$$
\begin{aligned}
x_{15 n-14}= & \frac{x_{15 n-29}}{1+x_{15 n-17} x_{15 n-20} x_{15 n-23} x_{15 n-26} x_{15 n-29}} \\
= & \frac{a_{14} \prod_{i=0}^{n-2}\left(\frac{1+\left(5 i+M_{14}-1\right) P_{14}}{1+\left(5 i+M_{14}\right) P_{14}}\right)}{1+\prod_{j=0}^{4}\left(a_{3 j+2} \prod_{i=0}^{n-2}\left(\frac{1+\left(5 i+M_{3 j+2}-1\right) P_{3 j+2}}{1+\left(5 i+M_{3 j+2}\right) P_{3 j+2}}\right)\right)}=\frac{a_{14} \prod_{i=0}^{n-2}\left(\frac{1+(5 i) a_{2} a_{5} a_{8} a_{11} a_{14}}{1+(5 i+1) a_{2} a_{5} a_{8} a_{11} a_{14}}\right)}{1+a_{2} a_{5} a_{8} a_{11} a_{14} \prod_{i=0}^{n-2}\left(\frac{1+(5 i) a_{2} a_{5} a_{8} a_{11} a_{14}}{1+(5 i+5) a_{2} a_{5} a_{8} a_{11} a_{14}}\right)} .
\end{aligned}
$$

Hence, we have

$$
x_{15 n-14}=a_{14} \prod_{i=0}^{n-1}\left(\frac{1+(5 i) a_{2} a_{5} a_{8} a_{11} a_{14}}{1+(5 i+1) a_{2} a_{5} a_{8} a_{11} a_{14}}\right) .
$$

Also, it follows from Eq. (2.1) and using Eq. (2.3) that

$$
\begin{aligned}
x_{15 n-13}= & \frac{x_{15 n-28}}{1+x_{15 n-16} x_{15 n-19} x_{15 n-22} x_{15 n-25} x_{15 n-28}} \\
= & \frac{a_{13} \prod_{i=0}^{n-2}\left(\frac{1+\left(5 i+M_{13}-1\right) P_{13}}{1+\left(5 i+M_{13}\right) P_{13}}\right)}{1+\prod_{j=0}^{4}\left(a_{3 j+1} \prod_{i=0}^{n-2}\left(\frac{1+\left(5 i+M_{3 j+1}-1\right) P_{3 j+1}}{1+\left(5 i+M_{3 j+1}\right) P_{3 j+1}}\right)\right)}=\frac{a_{13} \prod_{i=0}^{n-2}\left(\frac{1+(5 i) a_{1} a_{4} a_{7} a_{10} a_{13}}{1+(5 i+1) a_{1} a_{4} a_{7} a_{10} a_{13}}\right)}{1+a_{1} a_{4} a_{7} a_{10} a_{13} \prod_{i=0}^{n-2}\left(\frac{1+(5 i) a_{1} a_{4} a_{7} a_{10} a_{13}}{1+(5 i+5) a_{1} a_{4} a_{7} a_{10} a_{13}}\right)} .
\end{aligned}
$$


Hence, we have

$$
x_{15 n-13}=a_{13} \prod_{i=0}^{n-1}\left(\frac{1+(5 i) a_{1} a_{4} a_{7} a_{10} a_{13}}{1+(5 i+1) a_{1} a_{4} a_{7} a_{10} a_{13}}\right) .
$$

Also, it follows from Eq. (2.1) and using Eq. (2.3) that

$$
\begin{aligned}
x_{15 n-12} & =\frac{x_{15 n-27}}{1+x_{15 n-15} x_{15 n-18} x_{15 n-21} x_{15 n-24} x_{15 n-27}} \\
& =\frac{a_{12} \prod_{i=0}^{n-2}\left(\frac{1+\left(5 i+M_{12}-1\right) P_{12}}{1+\left(5 i+M_{12}\right) P_{12}}\right)}{1+\prod_{j=0}^{4}\left(a_{3 j} \prod_{i=0}^{n-2}\left(\frac{1+\left(5 i+M_{3 j}-1\right) P_{3 j}}{1+\left(5 i+M_{3 j}\right) P_{3 j}}\right)\right)}=\frac{a_{12} \prod_{i=0}^{n-2}\left(\frac{1+(5 i) a_{0} a_{3} a_{6} a_{9} a_{12}}{1+(5 i+1) a_{0} a_{3} a_{6} a_{9} a_{12}}\right)}{1+a_{0} a_{3} a_{6} a_{9} a_{12} \prod_{i=0}^{n-2}\left(\frac{1+(5 i) a_{0} a_{3} a_{6} a_{9} a_{12}}{1+(5 i+5) a_{0} a_{3} a_{6} a_{9} a_{12}}\right)} .
\end{aligned}
$$

Hence, we have

$$
x_{15 n-12}=a_{12} \prod_{i=0}^{n-1}\left(\frac{1+(5 i) a_{0} a_{3} a_{6} a_{9} a_{12}}{1+(5 i+1) a_{0} a_{3} a_{6} a_{9} a_{12}}\right) .
$$

Similarly, one can easily obtain the other relations for Eq. (2.2). Hence, the proof is completed.

Theorem 2.2. Assume that the initial values of the difference Eq. $(2.1) x_{-14}, x_{-13}, \ldots, x_{0} \in[0, \infty)$, then every solution of Eq. (2.1) is bounded.

Proof. Let $\left\{x_{\mathfrak{n}}\right\}_{\mathfrak{n}=-14}^{\infty}$ be a solution of Eq. (2.1). It follows from Eq. (2.1) that

$$
0 \leqslant x_{n+1}=\frac{x_{n-14}}{1+x_{n-2} x_{n-5} x_{n-8} x_{n-11} x_{n-14}} \leqslant x_{n-14} \text { for all } n \geqslant 0 .
$$

Then the sequence $\left\{x_{15 n-i}\right\}_{n=0}^{\infty}, i=0,1, \ldots, 14$ is decreasing and so is bounded from above by $M=$ $\max \left\{x_{-14}, x_{-13}, \ldots, x_{0}\right\}$.

Theorem 2.3. The only equilibrium point $\bar{x}$ of Eq. (2.1) is $\bar{x}=0$.

Proof. From Eq. (2.1), we can write $\bar{x}=\frac{\bar{x}}{1+\bar{x}^{5}}$. Then we have $\bar{x}+\bar{x}^{6}=\bar{x}$, or, $\bar{x}^{6}=0$. Thus, the only equilibrium point of Eq. (2.1) is $\bar{x}=0$.

Theorem 2.4. Assume that the initial values of the difference Eq. (2.1) $x_{-14}, x_{-13}, \ldots, x_{0} \in[0, \infty)$, then the equilibrium point $\bar{x}=0$ of Eq. (2.1) is locally stable.

Proof. Let $\epsilon>0$, and let $\left\{x_{n}\right\}_{n=-14}^{\infty}$ be a solution of Eq. (2.1) such that

$$
\sum_{j=0}^{14}\left|x_{-j}\right|<\epsilon
$$

It suffices to show that $\left|x_{1}\right|<\epsilon$. Now

$$
0<x_{1}=\frac{x_{-14}}{1+x_{-2} x_{-5} x_{-8} x_{-11} x_{-14}} \leqslant x_{-14}<\epsilon,
$$

and so the proof is completed.

Theorem 2.5. Assume that the initial values of the difference Eq. $(2.1) x_{-14}, x_{-13}, \ldots, x_{0} \in[0, \infty)$, then the equilibrium point $\bar{x}=0$ of Eq. (2.1) is globally asymptotically stable. 
Proof. We know by Theorem 2.4 that the equilibrium point $\bar{x}=0$ of Eq. (2.1) is locally stable. So let $\left\{x_{n}\right\}_{n=-14}^{\infty}$ be a positive solution of Eq. (2.1). It suffices to show that $\lim _{n \rightarrow \infty} x_{n}=\bar{x}=0$. From Theorem 2.2 we have $x_{n+1}<x_{n-14}$ for all $n \geqslant 0$, so the sequences $\left\{x_{15 n-i}\right\}_{n=0}^{\infty}, i=0,1, \ldots, 14$ are decreasing and bounded which implies that the sequences $\left\{x_{15 n-i}\right\}_{\mathfrak{n}=0}^{\infty}, i=0,1, \ldots, 14$ converge to limit (say $L_{i}>0$ ). So

$$
\mathrm{L}_{14}=\frac{\mathrm{L}_{14}}{1+\mathrm{L}_{2} \mathrm{~L}_{5} \mathrm{~L}_{8} \mathrm{~L}_{11} \mathrm{~L}_{14}}=0, \mathrm{~L}_{13}=\frac{\mathrm{L}_{13}}{1+\mathrm{L}_{1} \mathrm{~L}_{4} \mathrm{~L}_{7} \mathrm{~L}_{10} \mathrm{~L}_{13}}=0, \ldots, \mathrm{L}_{0}=\frac{\mathrm{L}_{0}}{1+\mathrm{L}_{0} \mathrm{~L}_{3} \mathrm{~L}_{6} \mathrm{~L}_{9} \mathrm{~L}_{12}}=0,
$$

which implies that $\mathrm{L}_{0}=\mathrm{L}_{1}=\cdots=\mathrm{L}_{14}=0$, from which the result follows.

\section{The difference equation $x_{n+1}=\frac{x_{n-14}}{1-x_{n-2} x_{n-5} x_{n-8} x_{n-11} x_{n-14}}$}

In this section we give a specific form of the solutions of the second equation in the form

$$
x_{n+1}=\frac{x_{n-14}}{1-x_{n-2} x_{n-5} x_{n-8} x_{n-11} x_{n-14}}, \quad n=0,1,2, \ldots,
$$

with conditions posed on the initial values $x_{-j}, j=0,1,2, \ldots, 14$.

Theorem 3.1. Let $\left\{x_{n}\right\}_{n=-14}^{\infty}$ be a solution of the difference Eq. (3.1). Then for $n=0,1,2, \ldots$,

$$
x_{15 n-k}=a_{k} \prod_{i=0}^{n-1}\left(\frac{-1+\left(5 i+M_{k}-1\right) P_{k}}{-1+\left(5 i+M_{k}\right) P_{k}}\right),
$$

where $P_{k}=\prod_{j=0}^{4} a_{\bmod (k, 3)+3 j}, M_{k}=5-\left[\frac{k}{3}\right]$ and $x_{-k}=a_{k}$, with $r_{k} \neq 1$ such that $r \in\{1,2,3, \ldots\}, k=$ $0,1,2, \ldots, 14$.

Proof. For $n=0$, the result holds. Now suppose that $n>0$ and that our assumption holds for $n-1$. That is

$$
\mathrm{x}_{15 n-15-k}=\mathrm{a}_{\mathrm{k}} \prod_{i=0}^{n-2}\left(\frac{-1+\left(5 i+M_{k}-1\right) \mathrm{P}_{k}}{-1+\left(5 i+M_{k}\right) \mathrm{P}_{k}}\right) .
$$

Now, it follows from Eq. (3.1) and using Eq. (3.3) that

$$
\begin{aligned}
x_{15 n-14} & =\frac{x_{15 n-29}}{1-x_{15 n-17} x_{15 n-20} x_{15 n-23} x_{15 n-26} x_{15 n-29}} \\
& =\frac{a_{14} \prod_{i=0}^{n-2}\left(\frac{-1+\left(5 i+M_{14}-1\right) P_{14}}{-1+\left(5 i+M_{14}\right) P_{14}}\right)}{1-\prod_{j=0}^{4}\left(a_{3 j+2} \prod_{i=0}^{n-2}\left(\frac{-1+\left(5 i+M_{3 j+2}-1\right) P_{3 j+2}}{-1+\left(5 i+M_{3 j+2}\right) P_{3 j+2}}\right)\right)}=\frac{a_{14} \prod_{i=0}^{n-2}\left(\frac{-1+(5 i) a_{2} a_{5} a_{8} a_{11} a_{14}}{-1+(5 i+1) a_{2} a_{5} a_{8} a_{11} a_{14}}\right)}{1-a_{2} a_{5} a_{8} a_{11} a_{14} \prod_{i=0}^{n-2}\left(\frac{-1+(5 i) a_{2} a_{5} a_{8} a_{11} a_{14}}{-1+(5 i+5) a_{2} a_{5} a_{8} a_{11} a_{14}}\right)} .
\end{aligned}
$$

Hence, we have

$$
x_{15 n-14}=a_{14} \prod_{i=0}^{n-1}\left(\frac{-1+(5 i) a_{2} a_{5} a_{8} a_{11} a_{14}}{-1+(5 i+1) a_{2} a_{5} a_{8} a_{11} a_{14}}\right) .
$$

Also, it follows from Eq. (3.1) and using Eq. (3.3) that

$$
\begin{aligned}
x_{15 n-13}= & \frac{x_{15 n-28}}{1-x_{15 n-16} x_{15 n-19} x_{15 n-22} x_{15 n-25} x_{15 n-28}} \\
= & \frac{a_{13} \prod_{i=0}^{n-2}\left(\frac{-1+\left(5 i+M_{13}-1\right) P_{13}}{-1+\left(5 i+M_{13}\right) P_{13}}\right)}{1-\prod_{j=0}^{4}\left(a_{3 j+1} \prod_{i=0}^{n-2}\left(\frac{-1+\left(5 i+M_{3 j+1}-1\right) P_{3 j+1}}{-1+\left(5 i+M_{3 j+1}\right) P_{3 j+1}}\right)\right)}=\frac{a_{13} \prod_{i=0}^{n-2}\left(\frac{-1+(5 i) a_{1} a_{4} a_{7} a_{10} a_{13}}{-1+(5 i+1) a_{1} a_{4} a_{7} a_{10} a_{13}}\right)}{1-a_{1} a_{4} a_{7} a_{10} a_{13} \prod_{i=0}^{n-2}\left(\frac{-1+(5 i) a_{1} a_{4} a_{7} a_{10} a_{13}}{-1+(5 i+5) a_{1} a_{4} a_{7} a_{10} a_{13}}\right)} .
\end{aligned}
$$


Hence, we have

$$
x_{15 n-13}=a_{13} \prod_{i=0}^{n-1}\left(\frac{-1+(5 i) a_{1} a_{4} a_{7} a_{10} a_{13}}{-1+(5 i+1) a_{1} a_{4} a_{7} a_{10} a_{13}}\right) .
$$

Also, it follows from Eq. (3.1) and using Eq. (3.3) that

$$
\begin{aligned}
x_{15 n-12}= & \frac{x_{15 n-27}}{1-x_{15 n-15} x_{15 n-18} x_{15 n-21} x_{15 n-24} x_{15 n-27}} \\
= & \frac{a_{12} \prod_{i=0}^{n-2}\left(\frac{-1+\left(5 i+M_{12}-1\right) P_{12}}{-1+\left(5 i+M_{12}\right) P_{12}}\right)}{1-\prod_{j=0}^{4}\left(a_{3 j} \prod_{i=0}^{n-2}\left(\frac{-1+\left(5 i+M_{3 j}-1\right) P_{3 j}}{-1+\left(5 i+M_{3 j}\right) P_{3 j}}\right)\right)}=\frac{a_{12} \prod_{i=0}^{n-2}\left(\frac{-1+(5 i) a_{0} a_{3} a_{6} a_{9} a_{12}}{-1+(5 i+1) a_{0} a_{3} a_{6} a_{9} a_{12}}\right)}{1-a_{0} a_{3} a_{6} a_{9} a_{12} \prod_{i=0}^{n-2}\left(\frac{-1+(5 i) a_{0} a_{3} a_{6} a_{9} a_{12}}{-1+(5 i+5) a_{0} a_{3} a_{6} a_{9} a_{12}}\right)} .
\end{aligned}
$$

Hence, we have

$$
x_{15 n-12}=a_{12} \prod_{i=0}^{n-1}\left(\frac{-1+(5 i) a_{0} a_{3} a_{6} a_{9} a_{12}}{-1+(5 i+1) a_{0} a_{3} a_{6} a_{9} a_{12}}\right) .
$$

Similarly, one can easily obtain the other relations for Eq. (3.2). Hence, the proof is completed.

Theorem 3.2. Eq. (3.1) has a unique equilibrium point $\bar{x}=0$, which is a non hyperbolic equilibrium point.

Proof. From Eq. (3.1), we can write $\bar{x}=\frac{\bar{x}}{1-\bar{x}^{5}}$. Then we have $\bar{x}-\bar{x}^{6}=\bar{x}$, or, $\bar{x}^{6}=0$. Thus the only equilibrium point of Eq. (3.1) is $\bar{x}=0$. Let $f:(0, \infty)^{5} \rightarrow(0, \infty)$ be a function defined by

$$
f(x, y, z, u, v)=\frac{x}{1-x y z u v} \text {. }
$$

Then we have

$$
\begin{array}{ll}
f_{x}(x, y, z, u, v)=\frac{1}{(1-x y z u v)^{2}}, & f_{y}(x, y, z, u, v)=\frac{x^{2} z u v}{(1-x y z u v)^{2}}, \\
f_{z}(x, y, z, u, v)=\frac{x^{2} y u v}{(1-x y z u v)^{2}}, & f_{u}(x, y, z, u, v)=\frac{x^{2} y z v}{(1-x y z u v)^{2}}, \\
f_{v}(x, y, z, u, v)=\frac{x^{2} y z u}{(1-x y z u v)^{2}} . &
\end{array}
$$

which implies that

$$
f_{x}(\bar{x}, \bar{x}, \bar{x}, \bar{x}, \bar{x})=1, f_{y}(\bar{x}, \bar{x}, \bar{x}, \bar{x}, \bar{x})=f_{z}(\bar{x}, \bar{x}, \bar{x}, \bar{x}, \bar{x})=f_{\mathfrak{u}}(\bar{x}, \bar{x}, \bar{x}, \bar{x}, \bar{x})=f_{v}(\bar{x}, \bar{x}, \bar{x}, \bar{x}, \bar{x})=0 .
$$

So, the linearized equation of Eq. (3.1) about the equilibrium point $\bar{x}=0$ is

$$
z_{\mathfrak{n}+1}=z_{\mathfrak{n}-14}
$$

and the characteristic equation of Eq. (3.4) about the equilibrium point $\bar{x}=0$ is

$$
\lambda^{15}-1=0,
$$

which implies that

$$
\left|\lambda_{i}\right|=1 ; i=1,2, \ldots, 15,
$$

so, $\bar{x}$ is a non hyperbolic equilibrium point.

Open Problem: Investigate the global behavior of solutions of Eq. (3.1) about the equilibrium point $\bar{x}=0$. 
4. The difference equation $x_{n+1}=\frac{x_{n-14}}{-1+x_{n-2} x_{n-5} x_{n-8} x_{n-11} x_{n-14}}$

In this section we give a specific form of the solutions of the third equation in the form

$$
x_{n+1}=\frac{x_{n-14}}{-1+x_{n-2} x_{n-5} x_{n-8} x_{n-11} x_{n-14}}, \quad n=0,1,2, \ldots,
$$

with conditions posed on the initial values $x_{-j}, j=0,1,2, \ldots, 14$. Also, we investigate the oscillation and periodicity of these solutions.

Theorem 4.1. Every solution of Eq. (4.1) is periodic with period 30.

Proof. From Eq. (4.1), we have

$$
x_{n+30}=\frac{x_{n+15}}{-1+\prod_{i=0}^{4} x_{n+15+3 i}} .
$$

Since

$$
\begin{aligned}
-1+\prod_{i=0}^{4} x_{n+15+3 i} & =-1+\prod_{i=0}^{3} x_{n+15+3 i} \frac{x_{n+12}}{-1+\prod_{i=0}^{4} x_{n+12+3 i}} \\
& =\frac{1}{-1+\prod_{i=0}^{4} x_{n+12+3 i}} \\
& \Rightarrow-1+\prod_{i=0}^{4} x_{n+15+3 i}=\frac{1}{-1+\prod_{i=0}^{4} x_{n+12+3 i}} .
\end{aligned}
$$

Similarly

$$
-1+\prod_{i=0}^{4} x_{n+12+3 i}=\frac{1}{-1+\prod_{i=0}^{4} x_{n+9+3 i}}
$$

so

$$
-1+\prod_{i=0}^{4} x_{n+15+3 i}=-1+\prod_{i=0}^{4} x_{n+9+3 i}
$$

Similarly

$$
-1+\prod_{i=0}^{4} x_{n+9+3 i}=-1+\prod_{i=0}^{4} x_{n+3+3 i} \text { and }-1+\prod_{i=0}^{4} x_{n+3+3 i}=\frac{1}{-1+\prod_{i=0}^{4} x_{n+3 i}},
$$

which imply from Eq. (4.1) and Eq. (4.2) that

$$
x_{n+30}=\frac{\left(\frac{x_{n}}{-1+\prod_{i=0}^{4} x_{n+3 i}}\right)}{\left(\frac{1}{-1+\prod_{i=0}^{4} x_{n+3 i}}\right)}=x_{n}, n=0,1,2, \ldots .
$$


Theorem 4.2. The periodic 30 solution of Eq. (4.1) has the form

$$
x_{30 n-k}=\frac{a_{k-(q(k))(15)^{q(k)}}}{\left(-1+p_{k-15}\right)^{\alpha(k) q(k)}} ; k=0,1, \ldots, 29 \text { and } n=1,2, \ldots,
$$

where $x_{-j}=a_{j}, p_{j}=\prod_{i=0}^{4} a_{\bmod (j, 3)+3 i}$, with $p_{j} \neq 1, j=0,1,2, \ldots, 14, p_{-l}=0, l=1,2, \ldots, 15, q(k)=$ $\frac{1}{2}\left((-1)^{\left[\frac{k}{15}\right]+1}+1\right)$ and $\alpha(k)=(-1)^{\left[\frac{k}{3}\right]+1}$.

Proof. From the definition of $q(k)$, we can see that

$$
\mathrm{q}(0)=\mathrm{q}(1)=\cdots=\mathrm{q}(14)=0 \text { and } \mathrm{q}(15)=\mathrm{q}(16)=\cdots=\mathrm{q}(29)=1 .
$$

Also,

$$
\alpha(i+6 r)=-1, \alpha(i+3+6 r)=1 ; i=0,1,2 \text { and } r=0,1,2,3,4
$$

So,

$$
\begin{aligned}
& x_{1}=\frac{x_{-14}}{-1+x_{-2} x_{-5} x_{-8} x_{-11} x_{-14}}=\frac{a_{14}}{-1+a_{2} a_{5} a_{8} a_{11} a_{14}}, \\
& x_{2}=\frac{x_{-13}}{-1+x_{-1} x_{-4} x_{-7} x_{-10} x_{-13}}=\frac{a_{13}}{-1+a_{1} a_{4} a_{7} a_{10} a_{13}}, \\
& x_{3}=\frac{x_{-12}}{-1+x_{0} x_{-3} x_{-6} x_{-9} x_{-12}}=\frac{a_{12}}{-1+a_{0} a_{3} a_{6} a_{9} a_{12}} \text {, } \\
& x_{5}=\frac{x_{-10}}{-1+x_{2} x_{-1} x_{-4} x_{-7} x_{-10}}=a_{10}\left(-1+a_{1} a_{4} a_{7} a_{10} a_{13}\right) \text {, } \\
& x_{7}=\frac{x_{-8}}{-1+x_{4} x_{1} x_{-2} x_{-5} x_{-8}}=\frac{a_{8}}{-1+a_{2} a_{5} a_{8} a_{11} a_{14}}, \\
& x_{9}=\frac{x_{-6}}{-1+x_{6} x_{3} x_{0} x_{-3} x_{-6}}=\frac{a_{6}}{-1+a_{0} a_{3} a_{6} a_{9} a_{12}}, \\
& x_{11}=\frac{x_{-4}}{-1+x_{8} x_{5} x_{2} x_{-1} x_{-4}}=a_{4}\left(-1+a_{1} a_{4} a_{7} a_{10} a_{13}\right) \text {, } \\
& x_{13}=\frac{x_{-2}}{-1+x_{10} x_{7} x_{4} x_{1} x_{-2}}=\frac{a_{2}}{-1+a_{2} a_{5} a_{8} a_{11} a_{14}}, \\
& x_{15}=\frac{x_{0}}{-1+x_{12} x_{9} x_{6} x_{3} x_{0}}=\frac{a_{0}}{-1+a_{0} a_{3} a_{6} a_{9} a_{12}} \text {, } \\
& x_{17}=\frac{x_{2}}{-1+x_{14} x_{11} x_{8} x_{5} x_{2}}=a_{13} \text {, } \\
& x_{19}=\frac{x_{4}}{-1+x_{16} x_{13} x_{10} x_{7} x_{4}}=a_{11} \text {, } \\
& x_{21}=\frac{x_{6}}{-1+x_{18} x_{15} x_{12} x_{9} x_{6}}=a_{9} \text {, } \\
& x_{23}=\frac{x_{8}}{-1+x_{20} x_{17} x_{14} x_{11} x_{8}}=a_{7} \text {, } \\
& x_{25}=\frac{x_{10}}{-1+x_{22} x_{19} x_{16} x_{13} x_{10}}=a_{5} \text {, } \\
& x_{27}=\frac{x_{12}}{-1+x_{24} x_{21} x_{18} x_{15} x_{12}}=a_{3} \text {, } \\
& x_{29}=\frac{x_{14}}{-1+x_{26} x_{23} x_{20} x_{17} x_{14}}=a_{1} \text {, } \\
& x_{4}=\frac{x_{-11}}{-1+x_{1} x_{-2} x_{-5} x_{-8} x_{-11}}=a_{11}\left(-1+a_{2} a_{5} a_{8} a_{11} a_{14}\right) \text {, } \\
& x_{6}=\frac{x_{-9}}{-1+x_{3} x_{0} x_{-3} x_{-6} x_{-9}}=a_{9}\left(-1+a_{0} a_{3} a_{6} a_{9} a_{12}\right) \text {, } \\
& x_{8}=\frac{x_{-7}}{-1+x_{5} x_{2} x_{-1} x_{-4} x_{-7}}=\frac{a_{7}}{-1+a_{1} a_{4} a_{7} a_{10} a_{13}}, \\
& x_{10}=\frac{x_{-5}}{-1+x_{7} x_{4} x_{1} x_{-2} x_{-5}}=a_{5}\left(-1+a_{2} a_{5} a_{8} a_{11} a_{14}\right) \text {, } \\
& x_{12}=\frac{x_{-3}}{-1+x_{9} x_{6} x_{3} x_{0} x_{-3}}=a_{3}\left(-1+a_{0} a_{3} a_{6} a_{9} a_{12}\right) \text {, } \\
& x_{14}=\frac{x_{-1}}{-1+x_{11} x_{8} x_{5} x_{2} x_{-1}}=\frac{a_{1}}{-1+a_{1} a_{4} a_{7} a_{10} a_{13}}, \\
& x_{16}=\frac{x_{1}}{-1+x_{13} x_{10} x_{7} x_{4} x_{1}}=a_{14} \text {, } \\
& x_{18}=\frac{x_{3}}{-1+x_{15} x_{12} x_{9} x_{6} x_{3}}=a_{12} \text {, } \\
& x_{20}=\frac{x_{5}}{-1+x_{17} x_{14} x_{11} x_{8} x_{5}}=a_{10} \text {, } \\
& x_{22}=\frac{x_{7}}{-1+x_{19} x_{16} x_{13} x_{10} x_{7}}=a_{8} \text {, } \\
& x_{24}=\frac{x_{9}}{-1+x_{21} x_{18} x_{15} x_{12} x_{9}}=a_{6} \text {, } \\
& x_{26}=\frac{x_{11}}{-1+x_{23} x_{20} x_{17} x_{14} x_{11}}=a_{4} \\
& x_{28}=\frac{x_{13}}{-1+x_{25} x_{22} x_{19} x_{16} x_{13}}=a_{2} \text {, } \\
& x_{30}=\frac{x_{15}}{-1+x_{27} x_{24} x_{21} x_{18} x_{15}}=a_{0} \text {, }
\end{aligned}
$$

and the result follows by induction.

Theorem 4.3. Eq. (4.1) has two equilibrium points 0 and $\sqrt[5]{2}$, which are non hyperbolic equilibrium points.

Proof. The proof is similar to the proof of Theorem 3.2, and will be omitted.

Theorem 4.4. Eq. (4.1) is periodic of period 15 iff $p_{k}=2 ; k=0,1,2$ and will take the form

$$
x_{15 n-k}=a_{k} ; k=0,1, \ldots, 14 \text { and } n=0,1,2, \ldots .
$$


Proof. The proof follows immediately from Theorem 4.2.

Theorem 4.5. Assume that $\mathrm{a}_{0}, \mathrm{a}_{1}, \ldots, \mathrm{a}_{14} \in(0,1)$. Then the solution $\left\{x_{\mathfrak{n}}\right\}_{\mathfrak{n}=-14}^{\infty}$ of the difference Eq. (4.1) oscillates about the equilibrium point $\bar{x}=0$, with positive semicycles of length 15 , and negative semicycles of length 15.

Proof. From Theorem 4.2, we have $x_{1}, x_{2}, \ldots, x_{15}<0$ and $x_{16}, x_{17}, \ldots, x_{30}>0$, and the result follows by induction.

\section{The difference equation $x_{n+1}=\frac{x_{n-14}}{-1-x_{n-2} x_{n-5} x_{n-8} x_{n-11} x_{n-14}}$}

In this section we give a specific form of the solutions of the fourth equation in the form

$$
x_{n+1}=\frac{x_{n-14}}{-1-x_{n-2} x_{n-5} x_{n-8} x_{n-11} x_{n-14}}, \quad n=0,1,2, \ldots,
$$

with conditions posed on the initial values $x_{-j}, j=0,1,2, \ldots, 14$. Also, we investigate the oscillation and periodicity of these solutions.

Theorem 5.1. Every solution of Eq. (5.1) is periodic with period 30.

Proof. The proof is similar to the proof of Theorem 4.1, and will be omitted.

Theorem 5.2. The periodic 30 solution of Eq. (5.1) has the form

$$
x_{30 n-k}=\frac{a_{k-(q(k))(15) q(k)}}{\left(-1-p_{k-15}\right)^{\alpha(k) q(k)}} ; k=0,1, \ldots, 29 \text { and } n=1,2, \ldots,
$$

where $x_{-j}=a_{j}, p_{j}=\prod_{i=0}^{4} a_{\bmod (j, 3)+3 i}$, with $p_{j} \neq-1, j=0,1,2, \ldots, 14, p_{-l}=0, l=1,2, \ldots, 15, q(k)=$ $\frac{1}{2}\left((-1)^{\left[\frac{k}{15}\right]+1}+1\right)$ and $\alpha(k)=(-1)^{\left[\frac{k}{3}\right]+1}$.

Proof. From the definition of $q(k)$, we can see that

$$
\mathrm{q}(0)=\mathrm{q}(1)=\cdots=\mathrm{q}(14)=0 \text { and } \mathrm{q}(15)=\mathrm{q}(16)=\cdots=\mathrm{q}(29)=1 .
$$

Also,

$$
\alpha(i+6 r)=-1, \alpha(i+3+6 r)=1 ; i=0,1,2 \text { and } r=0,1,2,3,4 \text {. }
$$

So,

$$
\begin{aligned}
& x_{1}=\frac{x_{-14}}{-1-x_{-2} x_{-5} x_{-8} x_{-11} x_{-14}}=-\frac{a_{14}}{1+a_{2} a_{5} a_{8} a_{11} a_{14}}, \\
& x_{3}=\frac{x_{-12}}{-1-x_{0} x_{-3} x_{-6} x_{-9} x_{-12}}=-\frac{a_{12}}{1+a_{0} a_{3} a_{6} a_{9} a_{12}}, \\
& x_{5}=\frac{x_{-10}}{-1-x_{2} x_{-1} x_{-4} x_{-7} x_{-10}}=-a_{10}\left(1+a_{1} a_{4} a_{7} a_{10} a_{13}\right), \\
& x_{7}=\frac{x_{-8}}{-1-x_{4} x_{1} x_{-2} x_{-5} x_{-8}}=-\frac{a_{8}}{1+a_{2} a_{5} a_{8} a_{11} a_{14}}, \\
& x_{9}=\frac{x_{6}}{-1-x_{6} x_{3} x_{0} x_{-3} x_{-6}}=\frac{x_{-4}}{-1+a_{0} a_{3} a_{6} a_{9} a_{12}}, \\
& x_{13}=\frac{x_{1}}{-1-x_{8} x_{5} x_{2} x_{-1} x_{-4}}=-a_{4}\left(1+a_{1} a_{4} a_{7} a_{10} a_{13}\right) \\
& x_{15}=\frac{x_{-2}}{-1-x_{10} x_{7} x_{4} x_{1} x_{-2}}=-\frac{a_{2}}{1+a_{2} a_{5} a_{8} a_{11} a_{14}},
\end{aligned}
$$

$$
\begin{aligned}
x_{2} & =\frac{x_{-13}}{-1-x_{-1} x_{-4} x_{-7} x_{-10} x_{-13}}=-\frac{a_{13}}{1+a_{1} a_{4} a_{7} a_{10} a_{13}}, \\
x_{4} & =\frac{x_{-11}}{-1-x_{1} x_{-2} x_{-5} x_{-8} x_{-11}}=-a_{11}\left(1+a_{2} a_{5} a_{8} a_{11} a_{14}\right), \\
x_{6} & =\frac{x_{-9}}{-1-x_{3} x_{0} x_{-3} x_{-6} x_{-9}}=-a_{9}\left(1+a_{0} a_{3} a_{6} a_{9} a_{12}\right), \\
x_{8} & =\frac{x_{-7}}{-1-x_{5} x_{2} x_{-1} x_{-4} x_{-7}}=-\frac{a_{7}}{1+a_{1} a_{4} a_{7} a_{10} a_{13}}, \\
x_{10} & =\frac{x_{-5}}{-1-x_{7} x_{4} x_{1} x_{-2} x_{-5}}=-a_{5}\left(1+a_{2} a_{5} a_{8} a_{11} a_{14}\right), \\
x_{12} & =\frac{x_{-3}}{-1-x_{9} x_{6} x_{3} x_{0} x_{-3}}=-a_{3}\left(1+a_{0} a_{3} a_{6} a_{9} a_{12}\right), \\
x_{14} & =\frac{x_{-1}}{-1-x_{11} x_{8} x_{5} x_{2} x_{-1}}=-\frac{a_{1}}{1+a_{1} a_{4} a_{7} a_{10} a_{13}}, \\
x_{16} & =\frac{x_{1}}{-1-x_{13} x_{10} x_{7} x_{4} x_{1}}=a_{14}
\end{aligned}
$$




$$
\begin{aligned}
& x_{17}=\frac{x_{2}}{-1-x_{14} x_{11} x_{8} x_{5} x_{2}}=a_{13}, \\
& x_{19}=\frac{x_{4}}{-1-x_{16} x_{13} x_{10} x_{7} x_{4}}=a_{11}, \\
& x_{21}=\frac{x_{6}}{-1-x_{18} x_{15} x_{12} x_{9} x_{6}}=a_{9}, \\
& x_{23}=\frac{x_{8}}{-1-x_{20} x_{17} x_{14} x_{11} x_{8}}=a_{7}, \\
& x_{25}=\frac{x_{10}}{-1-x_{22} x_{19} x_{16} x_{13} x_{10}}=a_{5} \\
& x_{27}=\frac{x_{12}}{-1-x_{24} x_{21} x_{18} x_{15} x_{12}}=a_{3} \\
& x_{29}=\frac{x_{14}}{-1-x_{26} x_{23} x_{20} x_{17} x_{14}}=a_{1},
\end{aligned}
$$

$$
\begin{aligned}
& x_{18}=\frac{x_{3}}{-1-x_{15} x_{12} x_{9} x_{6} x_{3}}=a_{12}, \\
& x_{20}=\frac{x_{5}}{-1-x_{17} x_{14} x_{11} x_{8} x_{5}}=a_{10}, \\
& x_{22}=\frac{x_{7}}{-1-x_{19} x_{16} x_{13} x_{10} x_{7}}=a_{8}, \\
& x_{24}=\frac{x_{9}}{-1-x_{21} x_{18} x_{15} x_{12} x_{9}}=a_{6}, \\
& x_{26}=\frac{x_{11}}{-1-x_{23} x_{20} x_{17} x_{14} x_{11}}=a_{4}, \\
& x_{28}=\frac{x_{13}}{-1-x_{25} x_{22} x_{19} x_{16} x_{13}}=a_{2}, \\
& x_{30}=\frac{x_{15}}{-1-x_{27} x_{24} x_{21} x_{18} x_{15}}=a_{0},
\end{aligned}
$$

and the result follows by induction.

Theorem 5.3. Eq. (5.1) has two equilibrium points 0 and $-\sqrt[5]{2}$, which are non hyperbolic equilibrium points.

Proof. The proof is similar to the proof of Theorem 3.2, and will be omitted.

Theorem 5.4. Eq. (5.1) is periodic of period 15 iff $p_{k}=-2 ; k=0,1,2$ and will be take the form

$$
x_{15 n-k}=a_{k} ; k=0,1, \ldots, 14 \text { and } n=0,1,2, \ldots .
$$

Proof. The proof follows immediately from Theorem 5.2.

Theorem 5.5. Assume that $\mathrm{a}_{0}, \mathrm{a}_{1}, \ldots, \mathrm{a}_{14} \in[0, \infty)$. Then the solution $\left\{\mathrm{x}_{\mathfrak{n}}\right\}_{\mathfrak{n}=-14}^{\infty}$ of the difference Eq. (5.1) oscillates about the equilibrium point $\bar{x}=0$, with positive semicycles of length 15 , and negative semicycles of length 15.

Proof. From Theorem 5.2, we have $x_{1}, x_{2}, \ldots, x_{15}<0$ and $x_{16}, x_{17}, \ldots, x_{30}>0$, and the result follows by induction.

\section{Numerical examples}

To verify the results of this paper, we consider some numerical examples as follows.

Example 6.1. The graph of the difference Eq. (2.1) and the case when $x_{-14}=7.8, x_{-13}=5.5, x_{-12}=7$, $x_{-11}=5, x_{-10}=9.8, x_{-9}=8.2, x_{-8}=7.9, x_{-7}=6.6, x_{-6}=1, x_{-5}=6, x_{-4}=3, x_{-3}=8.5, x_{-2}=2.9$, $x_{-1}=1.3$ and $x_{0}=6.9$ is shown in Figure 1.

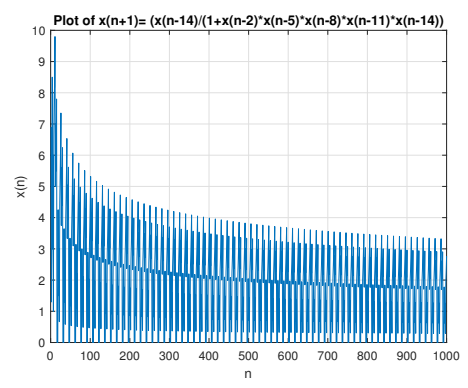

Figure 1: Graph of the difference Eq. (2.1).

Example 6.2. The graph of the difference Eq. (3.1) and the case when $x_{-14}=25, x_{-13}=16, x_{-12}=4$, $x_{-11}=9.5, x_{-10}=30, x_{-9}=10, x_{-8}=7.9, x_{-7}=6.6, x_{-6}=1.5, x_{-5}=6, x_{-4}=3, x_{-3}=5.5, x_{-2}=2$, $x_{-1}=1$ and $x_{0}=0.4$ is shown in Figure 2. 


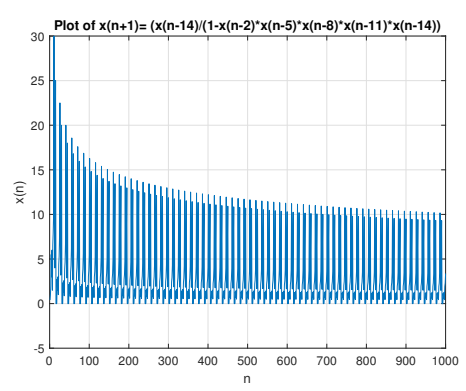

Figure 2: Graph of the difference Eq. (3.1).

Example 6.3. Figure 3 shows the period 30 solution and the oscillation of the solution of the difference Eq. (4.1) where $x_{-14}=0.15, x_{-13}=0.49, x_{-12}=0.85, x_{-11}=0.59, x_{-10}=0.25, x_{-9}=0.75, x_{-8}=0.8$, $x_{-7}=0.6, x_{-6}=0.5, x_{-5}=0.9, x_{-4}=0.4, x_{-3}=0.3, x_{-2}=0.7, x_{-1}=0.2$ and $x_{0}=0.1$.

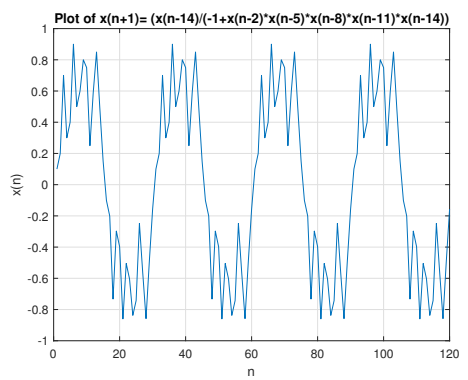

Figure 3: Graph of the difference Eq. (4.1).

Example 6.4. Figure 4 shows the period 30 solution and the oscillation of the solution of the difference Eq. (5.1) where $x_{-14}=0.8, x_{-13}=0.5, x_{-12}=2.5, x_{-11}=0.9, x_{-10}=0.5, x_{-9}=1, x_{-8}=1.2, x_{-7}=0.3$, $x_{-6}=0.22, x_{-5}=1.8, x_{-4}=0.85, x_{-3}=1.5, x_{-2}=2, x_{-1}=0.1$ and $x_{0}=0.3$.

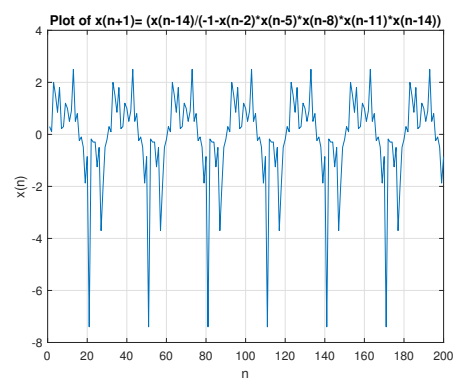

Figure 4: Graph of the difference Eq. (5.1).

\section{Acknowledgment}

The authors are grateful to the referees for numerous comments that improved the quality of the paper.

\section{References}

[1] R. P. Agarwal, Difference equations and inequalities, Marcel Dekker, New York, (2000). 1 
[2] R. P. Agarwal, E. M. Elsayed, Periodicity and stability of solutions of higher order rational difference equation, Adv. Stud. Contemp. Math., 17 (2008), 181-201.

[3] A. M. Ahmed, On the dynamics of a higher order rational difference equation, Discrete Dyn. Nat. Soc., 2011 (2011), 8 pages.

[4] A. M. Ahmed, H. El-Owaidy, A. E. Hamza, A. M. Youssef, On the recursive sequence $x_{n+1}=\frac{a+b x_{n-1}}{A+B x_{\mathfrak{n}}^{k}}$, J. Appl. Math. Inform., 27 (2009), 275-289. 1

[5] A. M. Ahmed, A. M. Youssef, A solution form of a class of higher-order rational difference equations, J. Egyptian Math. Soc., 21 (2013), 248-253. 1

[6] M. Aloqeili, Dynamics of a rational difference equation, Appl. Math. Comput., 176 (2006), 768-774. 1

[7] A. M. Amleh, J. Hoag, G. Ladas, A difference equation with eventually periodic solutions, Comput. Math. Appl., 36 (1998), 401-404. 1

[8] C. Çinar, On the positive solutions of the difference equation $x_{n+1}=\frac{x_{n-1}}{1+x_{n} x_{n-1}}$, Appl. Math. Comput., 150 (2004), 21-24. 1

[9] C. Çinar, On the difference equation $x_{n+1}=x_{n-1}-1+x_{n} x_{n-1}$, Appl. Math. Comput., 158 (2004), 813-816.

[10] C. Çinar, On the positive solutions of the difference equation $x_{n+1}=a x_{n-1} 1+b x_{n} x_{n-1}$, Appl. Math. Comput., 156 (2004), 587-590. 1

[11] R. Devault, V. L. Kocic, D. Stutson, Global behavior of solutions of the nonlinear difference equation $x_{n+1}=\frac{p_{n}+x_{n-1}}{x_{n}}$, , J. Difference Equ. Appl., 11 (2005), 707-719. 1

[12] E. M. Elsayed, On the difference equation $x_{n+1}=\frac{x_{n-5}}{-1+x_{n-2} x_{n-5}}$, Int. J. Contemp. Math. Sci., 3 (2008), 1657-1664. 1

[13] E. M. Elsayed, M. A. Marwa, Expressions and dynamical behavior of rational recursive sequences, J. Comput. Anal. Appl., 28 (2020), 67-78. 1

[14] E. A. Grove, G. Ladas, Periodicities in nonlinear difference equations, Chapman \& Hall/CRC, Boca Raton, (2005). 1

[15] R. Karatas, C. Cinar, D. Simsek, On positive solutions of the difference equation $x_{n+1}=\frac{x_{n-5}}{1+x_{n-2} x_{n-5}}$, Int. J. Contemp. Math. Sci., 1 (2006), 495-500.

[16] V. L. Kocić, G. Ladas, Global behavior of nonlinear difference equations of higher order with applications, Kluwer Academic Publishers, Dordrecht, (1993).

[17] M. R. S. Kulenović, G. Ladas, Dynamics of second order rational difference equations with open problems and conjectures, Chapman \& Hall/CRC, Florida, (2001). 1 